- Original Article

\title{
Effect of Coffee Consumption on the Progression of Type 2 Diabetes Mellitus among Prediabetic Individuals
}

\author{
Ji-Ho Lee, Mi-Kyeong Oh*, Jun-Tae Lim, Haa-Gyoung Kim, Won-Joon Lee \\ Department of Family Medicine, Gangneung Asan Hospital, University of Ulsan College of Medicine, Gangneung, Korea
}

\begin{abstract}
Background: A previous large-scale cohort study investigated the relationship between coffee intake and the progression of diabetes mellitus in the United States. However, studies on the effects of coffee on diabetes are rare in South Korea. Therefore, this study assessed the amount and method of coffee intake in Koreans in order to determine if coffee intake has a prophylactic effect on diabetes progression.

Methods: This study included 3,497 prediabetic patients from a single medical institution, with glycated hemoglobin levels ranging from $5.7 \%$ to $6.4 \%$. Cross-tabulation and Kaplan-Meier survival analyses were performed to compare patients with and without diabetes progression based on the frequency and method of coffee intake. Cox proportional hazard analysis was performed to correct for confounding variables.

Results: The observation period (mean \pm standard deviation) was $3.7 \pm 2.3$ years. Kaplan-Meier survival analysis revealed that the risk of diabetes progression was lowest in patients who drank black coffee three or more times per day $(\mathrm{P}=0.036)$. However, correction for confounding variables in Cox proportional hazard analysis revealed that, while the risk was lower for the patients who typically consumed black coffee than for those who mixed creamer and sugar into their coffees, the difference was not significant.

Conclusion: The results of this study suggest that drinking coffee without sugar and creamer at least three times daily has the greatest preventive effect on diabetes onset.
\end{abstract}

Keywords: Prediabetic State; Glycated Hemoglobin; Coffee; Diabetes Mellitus 


\section{INTRODUCTION}

Prediabetes is a strong risk factor of diabetes mellitus and cardiovascular diseases, and its incidence has been increasing in recent decades. Thus, many ongoing studies have evaluated risk factors associated with type 2 diabetes (diabetes). One risk factor identified in multimodal studies on favorite drinks was coffee intake. A study on the active components of coffee reported that chlorogenic acid, a strong antioxidant, might help regulate blood sugar levels and decrease the risk of diabetes. Chlorogenic acid inhibits intestinal glucose absorption and improves insulin sensitivity. However, few studies have been conducted on these active components. ${ }^{1)}$ Several studies and meta-analyses that analyzed the risk of diabetes and cardiovascular diseases have reported the beneficial effect of coffee intake. ${ }^{2)}$ Studies on the effect of coffee on cardiovascular diseases and lipid metabolism have also been conducted in South Korea. Similarly, studies on the effect of coffee preparation and consumption on blood lipid concentrations, ${ }^{3)}$ green coffee bean consumption on low density lipoprotein cholesterol oxidation inhibition, and glycogenesis reduction in overweight or obese women $^{4)}$ have also been reported. However, studies on the effect of coffee intake on diabetes progression are scarce.

In addition to the results of domestic and international studies on coffee intake, results from a large-scale prospective cohort study conducted in the United States were reported in April 2014. The results showed that participants who consumed one cup or more of black coffee per day had the lowest risk of diabetes progression, while those that drank less coffee had the highest risk of progression during the observation period. ${ }^{5)}$ Contrarily, a European study reported in May 2015 that coffee increased the risk of developing impaired fasting glucose in participants cytochrome P450 1A2 polymorphisms and hypertension. ${ }^{6)}$

However, studies on the effect of coffee on diabetes progression in Koreans are rare. This study analyzed the amount and method of coffee consumption in prediabetic patients who required active lifestyle modifications in order to assess the effects of coffee intake on glycated hemoglobin (HbAlc) levels.

\section{METHODS}

\section{Study Subjects}

This study utilized data from individuals undergoing health checkups at a single medical institution (health examination center of Gangneung Asan Hospital) from April 22, 2003 to June 30, $2014(n=6,246)$. Based on the prediabetes definition as $\mathrm{HbAlc}$ levels between $5.7 \%-6.4 \%$, 5,528 patients were categorized as prediabetic in the first examination. ${ }^{7,8)}$ Patients previously diagnosed with diabetes, who had a history of diabetes medication use, or who had only one measurement were ex- cluded from the study. Finally, a total of 3,497 patients were included in the analysis. Observation was terminated for patients with baseline $\mathrm{HbAlc}$ levels at or above $6.5 \%$. In addition, for non-diabetic cases, the last measurement was recorded as the final observation.

\section{Blood and Anthropometric Tests, Social History Investigation, and Variables}

Blood tests were performed after a fasting period starting at midnight of the night before. In all health examinations, the subjects wore the same hospital gowns and underwent measurements with the same method and same equipment by a designated examiner assigned to a specific area. Height was measured during full inhalation, with the subjects in bare feet or wearing only thin socks and standing on a horizontal surface with the heels, buttocks, back, and the back of the head lined up against a vertical surface. Measurements were taken from the top of the head and recorded to one decimal place. Weight and height were measured to one decimal place using a scale attached to a horizontal surface. Body mass index (BMI) was calculated as weight divided by the square of the height. Waist circumference was measured with the patient wearing a hospital gown and looking straight ahead, with both arms down. The tape measure was positioned parallel to the thinnest point, and the measurement was recorded to one decimal place. Blood pressure was taken manually after 30 minutes of rest by an examination center nurse; the same sphygmomanometer and stethoscope were used for all study participants. If the systolic pressure was $140 \mathrm{~mm} \mathrm{Hg}$ or higher, or the diastolic pressure was $80 \mathrm{~mm} \mathrm{Hg}$ or lower, the blood pressure measurement was repeated 30 minutes later and the new measurement recorded. Social history, including alcohol consumption, smoking status, and physical activity, was assessed based on responses to a selfadministered questionnaire.

Variables were selected and divided into subgroups according to standard type 2 diabetes screening test criteria ${ }^{9,10)}$ as well as a study on the prediction of diabetes in Koreans that used data from the national health nutritional analysis. ${ }^{11)}$ Korean criteria were used when the criteria differed from the American version. Diagnostic standards were based on HbAlc levels. According to American Diabetes Association criteria, prediabetic patients were defined as those with HbAlc levels of 5.7\%-6.4\%; $5.7 \%-5.8 \%$ indicated low risk, 5.9\%-6.0\% indicated moderate risk, and $6.1 \%-6.4 \%$ indicated high risk. HbAlc levels of $6.5 \%$ or higher were considered to be indicative of overt diabetes.

The patients were also divided according to smoking status: smokers and non-smokers, including ex-smokers. Group 1 included men with waist circumferences $<90 \mathrm{~cm}$ and women with waist circumferences $<85 \mathrm{~cm}$; group 2 included men with waist circumferences $\geq 90 \mathrm{~cm}$ and women with waist circumferences $\geq 85 \mathrm{~cm}$. The subjects were also divided according to 
blood pressure, including: diastolic blood pressure $<90 \mathrm{~mm}$ $\mathrm{Hg}$ and systolic blood pressure $<140 \mathrm{~mm} \mathrm{Hg}$, and diastolic blood pressure $\geq 90 \mathrm{~mm} \mathrm{Hg}$ and systolic pressure $\geq 140 \mathrm{~mm} \mathrm{Hg}$. Alcohol consumption was divided as follows, with $13 \mathrm{~g}$ of alcohol defined as one glass: < 13 g per week, 13-64 g per week, and $\geq 65$ g per week. BMIs were divided into $>$ or $<25 \mathrm{~kg} / \mathrm{m}^{2}$ and $\geq 25 \mathrm{~kg} / \mathrm{m}^{2}$. Triglyceride and high density lipoprotein (HDL) cholesterol levels were divided into $<$ and $\geq 250 \mathrm{mg} / \mathrm{dL}$ and $<$ and $\geq 35 \mathrm{mg} / \mathrm{dL}$, respectively. Age, sex, personal history of hypertension, familial history of hypertension and diabetes, and physical activity were also recorded. The questionnaire used in our hospital defined physical activity as light (office work, <2 hours of walking per day, or small amount of housework), normal (manufacturing, service industry, or 2-4 hours of walking per day, or large amount of housework), and heavy (farming, fishing, engineering, or construction work). The subjects selfreported their activity levels.

\section{Frequency and Method of Coffee Consumption}

The nutritional questionnaire in this study was a food frequency questionnaire. The consumption frequencies of black coffee and coffee with sugar and creamer were self-reported by the participants. Subgroups were further divided according to the consumption frequency of black coffee and coffee with sugar and creamer as well as the method of coffee consumption (Table 1). The consumption frequencies of black coffee and coffee with sugar and creamer were divided into more than three times per day, twice a day, once a day, five to six times per week, three to four times per week, one or two times per week, two or three times per month, and once or less per month. Subjects who reported drinking coffee $\leq 5$ or six times per week, as well as those who drank coffee once a day were placed in the 'once or less a day' group. The frequency of coffee consumption and preparation method were defined as follows.

First, the frequency of coffee consumption, regardless of the number of times sugar and creamer were added, were divided into the 'once or less a day' (1), 'twice a day' (2), and ' $\geq$ three

Table 1. Coffee preparation

\begin{tabular}{lccc}
\hline $\begin{array}{l}\text { Coffee } \\
\text { preference }\end{array}$ & $\begin{array}{c}\text { Daily no. of } \\
\text { coffees }\end{array}$ & Daily addition of creamer or sugar & No. (\%) \\
\hline A & $\leq 1$ & $\begin{array}{c}\text { Regardless of no. of creamer or sugar } \\
\text { added to coffee }\end{array}$ & $1,951(55.8)$ \\
B2 & 2 & $\begin{array}{c}\text { Both creamer and sugar added less } \\
\text { than once daily }\end{array}$ & $103(2.9)$ \\
B3 & $\geq 3$ & Creamer or sugar added more than & $143(4.1)$ \\
M2 & 2 & twice daily & $461(13.2)$ \\
M3 & $\geq 3$ & & $839(24.0)$ \\
\hline
\end{tabular}

${ }^{*} \mathrm{~A}$ : $\leq$ one coffee per day; B3: more than three coffees per day, less than one time with creamer and sugar; B2: two coffees per day, less than one time with creamer and sugar; M3: $\geq$ three coffees per day with sugar or creamer added twice; M2: two coffees per day with sugar or creamer added more than two times. times per day' groups (3). Second, the methods of coffee consumption were defined as follows: non-drinkers were defined as subjects who drank coffee once or less per day (A), regardless of the number times that sugar and creamer were added; the 'black coffee preferred twice a day' group: those who drank black coffee $\geq$ two times a day, with sugar or creamer added once or less (B2); the 'black coffee preferred three times per day' group: those who drank black coffee $\geq$ three times per day with sugar or creamer addition added $\leq$ one time (B3); the 'mixed coffee preferred twice a day' group: those who drank a coffee mixed with creamer and sugar $\geq$ two times per day with sugar or creamer added $\geq$ two times (M2); and the 'mixed coffee preferred three times per day' group: those who drank mixed coffee $\geq$ three times per day with sugar or creamer added $\geq$ two times (M3).

\section{Statistical Analysis}

SPSS Statistics for Windows ver. 17.0 (SPSS Inc., Chicago, IL, USA) was used for statistical analysis. Among 3,497 subjects with baseline HbAlc levels between $5.7 \%$ and $6.4 \%$, those with subsequent levels $\geq 6.5 \%$ were allocated to the diabetes progression group, while those with lower values in the final measurement were allocated to the diabetes non-progression group. Cross-tabulation analysis was performed to analyze the effect of coffee consumption frequency, method, and other variables on diabetes progression. The analyzed variables included sex, age, waist circumference, systolic and diastolic blood pressures, alcohol consumption, smoking status, amount of physical activity, frequency of sugar and creamer consumption, BMI, triglyceride level, and HDL cholesterol levels. The trend test approximation was also measured for coffee consumption frequency, an ordinal variable, by using linear-by-linear association.

Kaplan-Meier survival analysis was performed for diabetes progression according to coffee consumption frequency and preparation. The variables that significantly affected diabetes progression and the known risk factors identified by previous publications were designated as confounding factors in the cross-tabulation analysis, while Cox proportional hazard analysis was used for correction.

\section{RESULTS}

The observation period (mean \pm standard deviation) was $3.7 \pm$ 2.3 years. The characteristics and baseline values of the 3,497 subjects are shown in Table 2. The mean frequency of coffee consumption $1.6 \pm 0.8$ times per day, and the mean number of sugar and creamer additions per day was $1.5 \pm 0.7$ times for both. Of the subjects, 1,951 (55.8\%) drank coffee once or less a day, $982(28.1 \%)$ drank coffee twice a day, and 564 (16.1\%) drank coffee $\geq 3$ times per day.

Cross-tabulation analysis revealed that 390 subjects (11.1\%) 
Table 2. General study population characteristics at baseline $(n=3,497)$

\begin{tabular}{lc}
\hline Characteristic & Value \\
\hline Sex (female) & $1,152(32.9)$ \\
Age (y) & $46.1 \pm 8.5$ \\
Medical history & \\
History of hypertension & $753(21.5)$ \\
Family history of hypertension & $902(25.8)$ \\
Family history of diabetes mellitus & $839(24.0)$ \\
Anthropometric data & \\
Waist measurement (cm) & $84.2 \pm 15.6$ \\
Diastolic BP (mm Hg) & $81.4 \pm 10.8$ \\
Systolic BP (mm Hg) & $125.4 \pm 15.1$ \\
Body mass index (kg/m²) & $24.8 \pm 3.1$ \\
Coffee, sugar, and creamer intake & \\
No. of coffees consumed daily & $1.6 \pm 0.8$ \\
No. of times sugar added to daily coffee & $1.5 \pm 0.7$ \\
No. of times creamer added to daily coffee & $1.5 \pm 0.7$ \\
Social history & \\
Current smoker & $697(19.9)$ \\
Physical activities & \\
$\quad$ Light & $1,807(51.7)$ \\
Moderate & $1,326(37.9)$ \\
$\quad$ High & $364(10.4)$ \\
Alcohol intake (g/wk) & $21.6 \pm 30.1$ \\
Glycated hemoglobin (\%) & $5.9 \pm 0.2$ \\
Lipid profile & \\
Total cholesterol (mg/dL) & \\
Triglyceride (mg/dL) & $188.2 \pm 33.3$ \\
HDL cholesterol (mg/dL) & $139.9 \pm 98.6$ \\
LDL cholesterol (mg/dL) & $120.1 \pm 31.9$ \\
\hline
\end{tabular}

Values are presented as number (\%) or mean \pm standard deviation.

$\mathrm{BP}$, blood pressure; DM, diabetes mellitus.

progressed to diabetes, including 89 (4.5\%) low-risk subjects with baseline HbAlc levels of 5.7\%-5.8\%, 122 (12.7\%) mid-risk subjects with $\mathrm{HbAlc}$ levels of 5.9\%-6.0\%, and 179 (32.8\%) highrisk subjects with HbAlc levels of $6.1 \%-6.4 \%$. These findings indicate that higher prediabetic risk levels were associated with higher incidence of diabetes progression. In addition, the variables known as diabetes risk factors all were significantly related to diabetes in the cross-tabulation analysis, except for family history. Diabetes progression was observed in $9.5 \%$ and $10.8 \%$ of subjects who consumed coffee twice and $\geq$ three times per day, respectively. Both values were lower than the $12.1 \%$ who consumed coffee once or less per day, although the difference was not statistically significant. Of the many methods of coffee consumption, those who preferred black coffee (B3 and B2) and those who mixed their coffee (M2 and M3) had fewer subjects who progressed to diabetes than the non-coffee preference group (A), but the difference was not significant (Table 3).

Kaplan-Meier survival analysis of the frequency of coffee consumption revealed that the probability of not progressing to diabetes (cumulative survival) was highest in the following order: $\geq$ three times per day, once or less, and twice a day (three $>$ one $>$ two). However, the $95 \%$ confidence intervals of these results were not statistically significant $(\mathrm{P}=0.922)$ (Figure 1). Among methods of coffee consumption, the probability of not progress-
Table 3. New onset of DM according to coffee intake

\begin{tabular}{lrcc}
\hline Variable & Total $(n=3,497)$ & DM $(n=390)$ & P-value \\
\hline $\begin{array}{l}\text { Glycated hemoglobin (\%) } \\
\text { Low (5.7-5.8) }\end{array}$ & $1,984(100.0)$ & $89(4.5)$ & $<0.001^{*}$ \\
Medium (5.9-6.0) & $958(100.0)$ & $122(12.7)$ & \\
$\quad$ High (6.1-6.4) & $552(100.0)$ & $179(32.4)$ & \\
No. of coffees consumed & & & $0.15^{*}$ \\
$\leq 1 /$ d & $1,951(100.0)$ & $236(12.1)$ & \\
2/d & $982(100.0)$ & $93(9.5)$ & \\
$\geq 3 /$ d & $564(100.0)$ & $61(10.8)$ & \\
Coffee preparation ${ }^{\dagger}$ & & & \\
A & $1,951(100.0)$ & $236(12.1)$ & \\
B3 & $103(100.0)$ & $10(9.7)$ & \\
B2 & $143(100.0)$ & $11(10.7)$ & \\
M3 & $461(100.0)$ & $51(11.1)$ & \\
M2 & $839(100.0)$ & $82(9.8)$ & \\
\hline
\end{tabular}

Values are presented as number (\%).

DM, diabetes mellitus.

*Linear by linear associations were used to evaluate trends in ordinal variables. ${ }^{\dagger} \mathrm{A}$ : $\leq$ one coffee per day; B3: more than three coffees per with creamer and sugar less than once per day; B2: coffee twice per day with creamer and sugar less than once per day; M3: $\geq$ three coffees per day with sugar or creamer more than twice daily; M2: two coffees per with sugar or creamer more than twice daily.

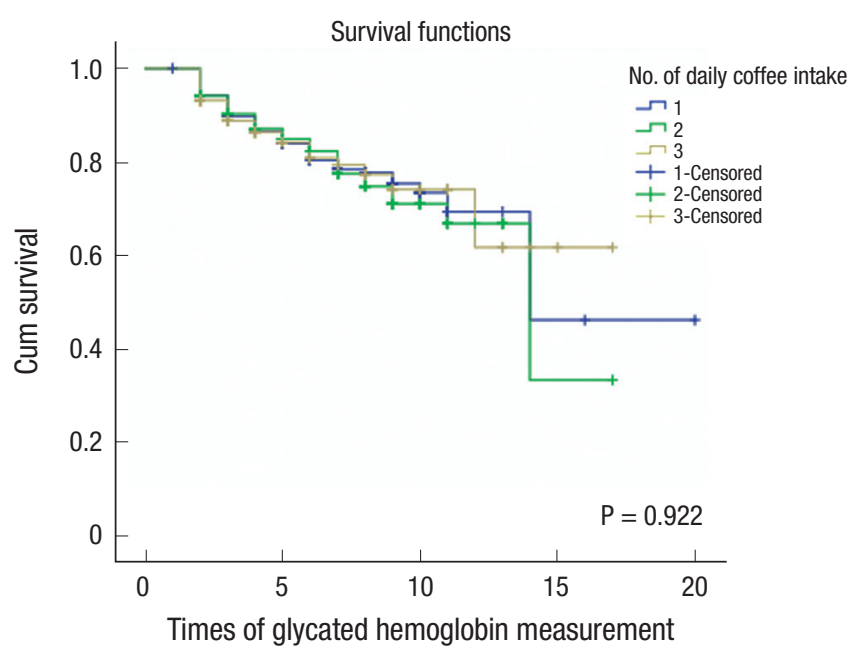

Figure 1. Kaplan-Meier curve of the daily number of coffees consumed. $1: \leq 0 n e / d, 2$ : two/d, 3: $\geq$ three/d.

ing to diabetes was highest in those who preferred to drink black coffee three or times more per (B3); those who preferred mixed coffee mix twice a day (M2) showed a lower probability of not progressing to diabetes than the those who did not consume coffee (A). Thus, the probability of not progressing to diabetes was highest in the $\mathrm{B} 3$ group, followed in order of probability by $\mathrm{B} 2, \mathrm{M} 3, \mathrm{~A}$, and M2, a statistically significant difference $(\mathrm{P}=0.036)$ (Figure 2).

Log-rank analysis of the method of coffee consumption revealed that the 'non-coffee preferred' (A) and 'black coffee preferred $\geq 3$ times per day' groups (B3) had a P-value of 0.069. Meanwhile, the 'once or less a day' (A) and 'coffee mix preferred $\geq$ threes times per day' group (M3) had a P-value of 0.072. While these values are not significant in the $95 \%$ confidence interval, they 


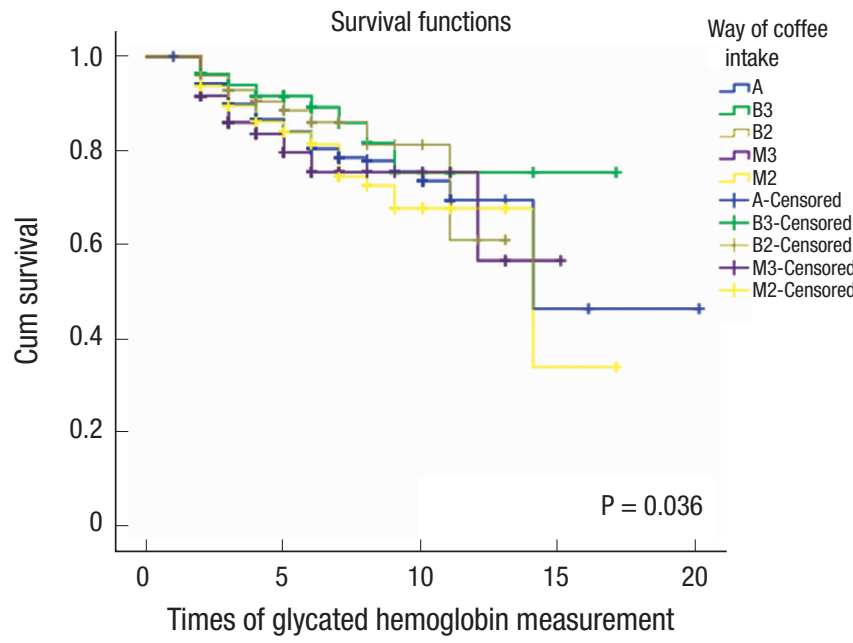

Figure 2. Kaplan-Meier curve for coffee preparation. A: $\leq$ one coffee per day, B3: more than three coffees per day of coffee, less than one time with creamer and sugar; B2: two coffees per day, less than one time with creamer and sugar; M3: $\geq$ three coffees per day with sugar or creamer more than twice a day; M2: coffee twice per day with sugar or creamer more than two times per day.

are significant in the $90 \%$ confidence interval. Of note, the 'black coffee preferred $\geq$ three times per day' and mixed coffee preferred groups (B3 and M3, respectively) showed a P-value of 0.010 , which is significantly different from the $95 \%$ confidence interval (CI). The log-rank analyses that compared the other consumption methods did not reveal statistically significant results.

Cox proportional hazard analysis corrected for covariates of the above-mentioned frequency of coffee consumption and methods revealed that consuming coffee $\geq$ two times a day was associated with a lower risk of diabetes progression than that associated with consuming coffee once per day or less consumption; however, the difference was not statistically significant. The hazard ratios among those who preferred black coffee preferred three times per day and twice per day were $0.92(\mathrm{P}=0.51$; 95\% CI, 0.72-1.18) and 0.71 ( $\mathrm{P}=0.19 ; 95 \% \mathrm{CI}, 0.42-1.19)$, respectively, which shows a lower risk of diabetes progression than in those who preferred coffee mixed with creamer and sugar and those who did not prefer to drink coffee, but the differences were also not statistically significant (Tables 4,5 ).

\section{DISCUSSION}

The Kaplan-Meier survival analysis results in this study indicate that consumption of coffee twice or more per day with no added sugar or creamer has a preventive effect on diabetes. However, Cox analysis with correction for confounding factors, including known risk factors in prediabetic patients such as age, BMI, family history of diabetes, hypertension, HDL cholesterol level, triglyceride level, smoking status, alcohol consumption, level of physical activity, and family history of hypertension, which were statistically significant in cross-tabulation
Table 4. Association between coffee intake and new onset of DM in Cox proportional hazard analysis

\begin{tabular}{|c|c|c|c|c|c|}
\hline \multirow{2}{*}{ Factor } & \multirow{2}{*}{ No. } & \multirow{2}{*}{$\begin{array}{l}\text { No. of new } \\
\text { DM onset }\end{array}$} & \multirow{2}{*}{$\begin{array}{c}\text { Hazard } \\
\text { ratio }\end{array}$} & \multicolumn{2}{|c|}{ 95\% Confidence interval } \\
\hline & & & & Lower & Upper \\
\hline \multicolumn{6}{|c|}{ No. of coffees consumed ${ }^{*}$} \\
\hline$\leq 1 / d$ & 1,951 & 236 & 1 & \multicolumn{2}{|c|}{ Reference } \\
\hline $2 / d$ & 982 & 93 & 0.88 & 0.67 & 1.16 \\
\hline$\geq 3 / d$ & 564 & 61 & 0.91 & 0.70 & 1.22 \\
\hline \multicolumn{6}{|c|}{ Coffee preparation ${ }^{\dagger}$} \\
\hline$A$ & 1,951 & 236 & 1 & \multicolumn{2}{|c|}{ Reference } \\
\hline B3 & 103 & 10 & 0.92 & 0.72 & 1.18 \\
\hline B2 & 143 & 11 & 0.71 & 0.42 & 1.19 \\
\hline M3 & 461 & 51 & 0.77 & 0.47 & 1.26 \\
\hline M2 & 839 & 82 & 1.22 & 0.88 & 1.70 \\
\hline
\end{tabular}

\section{DM, diabetes mellitus.}

*Cox regression for number of coffees consumed. Adjusted for sex, waist measurement, diastolic blood pressure, systolic blood pressure, alcohol intake, history of hypertension, family history of hypertension, family history of diabetes mellitus, body mass index, and triglyceride and high density lipoprotein cholesterol levels. ${ }^{\dagger}$ Cox regression for coffee preparation. Adjusted as for the number of coffees consumed.

Table 5. Association between coffee consumption and newly onset DM in Cox proportional hazard analysis with all confounding factors

\begin{tabular}{|c|c|c|c|c|c|}
\hline \multirow{2}{*}{ Factor } & \multirow{2}{*}{ No. } & \multirow{2}{*}{$\begin{array}{l}\text { No. of new } \\
\text { DM onset }\end{array}$} & \multirow{2}{*}{$\begin{array}{c}\text { Hazard } \\
\text { ratio }\end{array}$} & \multicolumn{2}{|c|}{ 95\% Confidence interval } \\
\hline & & & & Lower & Upper \\
\hline \multicolumn{6}{|c|}{ No. of coffees consumed ${ }^{*}$} \\
\hline$\leq 1 / d$ & 1,951 & 236 & 1 & \multicolumn{2}{|c|}{ Reference } \\
\hline $2 / d$ & 982 & 93 & 0.89 & 0.67 & 1.20 \\
\hline$\geq 3 / d$ & 564 & 61 & 0.91 & 0.59 & 1.12 \\
\hline \multicolumn{6}{|c|}{ Coffee preparation ${ }^{\dagger}$} \\
\hline A & 1,951 & 236 & 1 & \multicolumn{2}{|c|}{ Reference } \\
\hline B3 & 103 & 10 & 1.07 & 0.82 & 1.40 \\
\hline B2 & 143 & 11 & 0.95 & 0.55 & 1.63 \\
\hline M3 & 461 & 51 & 0.86 & 0.49 & 1.49 \\
\hline M2 & 839 & 82 & 1.32 & 0.92 & 1.90 \\
\hline
\end{tabular}

DM, diabetes mellitus.

${ }^{*}$ Cox regression for number of coffees consumed. Adjusted for sex, waist measurement, diastolic blood pressure, systolic blood pressure, alcohol intake, history of hypertension, family history of hypertension, family history of diabetes mellitus, body mass index, and triglyceride and high density lipoprotein cholesterol levels with age, physical activity, and smoking status. ${ }^{\dagger}$ Cox regression for coffee preparation. Adjusted as for number of coffees consumed.

analysis, did not reveal a significant association between frequency or method of coffee consumption and diabetes progression (Tables 4,5 ). The amount of physical activity was not surveyed in the questionnaire administered to patients in the health examination center of our hospital. Therefore, it was only included as a risk factor in the analysis. Future development of a tool that can quantify physical activity would be a useful addition to the questionnaire currently used in the health examination center of our hospital.

Contrary to the large-scale cohort study conducted in the United States that analyzed risks according to changes in coffee consumption, this study performed the analysis based on the baseline measurement. Therefore, additional confirmation was necessary to determine if there were changes in the frequencies of consumption of black coffee and coffee with sugar 
and creamer between the first and last measurements during the observation period. A paired t-test of the last HbAlc measurement revealed that the consumption frequencies of black coffee, coffee with sugar, and coffee with creamer were $1.7 \pm 0.8$ $(\mathrm{P}=0.001), 1.5 \pm 0.7(\mathrm{P}=0.912)$, and $1.6 \pm 0.8(\mathrm{P}=0.763)$, respectively, showing almost no change from the baseline frequencies. Thus, we were able to calculate the risk based on coffee consumption frequency and preparation at the baseline measurement of $\mathrm{HbAlc}$ levels. However, future studies might investigate the change in risk factors and amount of coffee consumption.

With the questionnaire used in the health examination center of our hospital made it difficult to determine the actual amount of coffee that was consumed. Thus, the analysis instead considered the number of times that coffee was consumed per day. Based on the American study results, 8 oz., 1.5 cups, or about $360 \mathrm{~mL}$ of coffee is recommended to prevent diabetes onset. This volume amounts to about $\geq 3$ cups based on the average cup size of 100-120 mL in Korea. ${ }^{12,13)}$ The subjects in this study consumed much less coffee that Europeans or Americans. Among those that consumed coffee twice or more a day, only $7 \%$ consumed black coffee, while $27 \%$ consumed coffee mixed with creamer and sugar. Of these subjects, only $4.2 \%$ consumed black coffee; $24 \%$ mixed their coffee with creamer and/or sugar $\geq$ three times per day, which is equivalent to one cup in the American study. The large differences between groups may have limited the statistical analysis findings. A randomized study with a controlled number of subjects per group is necessary to evaluate these findings.

The prophylactic effects of coffee intake on diabetes, as reported in the large-scale cohort study conducted in the United States, remain controversial. ${ }^{14-16)}$ More studies are needed to determine whether these observations are due to the effects of chlorogenic acid alone on glucose and lipid metabolism, increased weight and waist circumference due to increased consumption of sugar and creamer, or some other mechanism of active component inhibition. Moreover, as observed in a recent European study, personal diabetes risk factors such as genotype must also be considered when determining the effect of coffee intake on glucose regulation.

In this context, this study, though only reflecting the characteristics of residents of eastern Gangwon and Gangneung, determined the coffee consumption frequencies and methods (addition of sugar and creamer) of prediabetic Korean subjects as well as their effects on diabetes progression. ${ }^{17-19)}$ Additional studies are necessary to investigate the effects of coffee consumption and habits, including amount, method, and duration, on diabetes progression that also consider the unique characteristics of Koreans.

\section{CONFLICT OF INTEREST}

No potential conflict of interest relevant to this article was reported.

\section{REFERENCES}

1. Kim SH. Coffee: more knowledge, more taste. Korean Clin Diabetes 2009;10:250-4.

2. Hitman GA. Coffee: friend or foe for diabetes risk? Diabet Med 2014; 31:763.

3. Kim EK, Choe JS, Kim EK. Correlation of nutrient intake, obesity-related anthropometrics, and blood lipid status with instant coffee-mix intakes in Gangneung and Samcheok residents. Korean J Community Nutr 2013;18:134-41.

4. Park JY, Kim JY, Lee SP, Lee JH. The effect of green coffee bean extract supplementation on body fat reduction in overweight/obese women. Korean J Nutr 2010;43:374-81.

5. Bhupathiraju SN, Pan A, Manson JE, Willett WC, van Dam RM, Hu FB. Changes in coffee intake and subsequent risk of type 2 diabetes: three large cohorts of US men and women. Diabetologia 2014;57:1346-54.

6. Palatini P, Benetti E, Mos L, Garavelli G, Mazzer A, Cozzio S, et al. Association of coffee consumption and CYP1A2 polymorphism with risk of impaired fasting glucose in hypertensive patients. Eur J Epidemiol 2015; 30:209-17.

7. Tuso P. Prediabetes and lifestyle modification: time to prevent a preventable disease. Perm J 2014;18:88-93.

8. American Diabetes Association. Diagnosis and classification of diabetes mellitus. Diabetes Care 2010;33 Suppl 1:S62-9.

9. Korean Academy of Medical Sciences. Fact sheet: evidence-based recommendations for type 2 diabetes in primary care [Internet]. Seoul: Korean Academy of Medical Sciences [cited 2014 Jul 14]. Available from: http://www.kams.or.kr.

10. Ochoa PS, Terrell BT, Vega JA, Mnjoyan SZ, Lu C, Klein MS, et al. Identification of previously undiagnosed diabetes and prediabetes in the inpatient setting using risk factor and hemoglobin AlC screening. Ann Pharmacother 2014;48:1434-9.

11. Lee YH, Kim DJ. Diabetes risk score for Korean adults. J Korean Diabetes 2013;14:6-10.

12. Korean Ministry of Food and Drug Safety. Fact sheet: what is the level of caffeine intake of Korean?: evaluation of the capita intake of caffeine per day [Internet]. Cheongju: Korean Ministry of Food and Drug Safety; 2013 [cited 2014 Aug 6]. Available from: http://www.kdi.re.kr/infor/ ep_view.jsp?num=128460.

13. Fact sheet: market report for domestic processed food products of coffee [Internet]. Naju: Korea Agro-Fisheries \& Food Trade Corporation; 2013 [cited 2014 May 6]. Available from: http://www.aTFIS.or.kr.

14. Yamaji T, Mizoue T, Tabata S, Ogawa S, Yamaguchi K, Shimizu E, et al. Coffee consumption and glucose tolerance status in middle-aged Japanese men. Diabetologia 2004;47:2145-51.

15. Ding M, Bhupathiraju SN, Chen M, van Dam RM, Hu FB. Caffeinated and decaffeinated coffee consumption and risk of type 2 diabetes: a systematic review and a dose-response meta-analysis. Diabetes Care 2014;37:569-86. 
16. Pham NM, Nanri A, Kochi T, Kuwahara K, Tsuruoka H, Kurotani K, et al. Coffee and green tea consumption is associated with insulin resistance in Japanese adults. Metabolism 2014;63:400-8.

17. Chung SK, Kim JE, Kim GE. Association between coffee drinking and tumor-markers. Korean J Fam Pract 2014;4:56-60.

18. Park YM, Song YM, Choi J. Associations between irritable bowel syn- drome and psychological factors in Korean high-school girls. Korean J Fam Pract 2011;1:111-20.

19. Lee SJ, Song YM, Park JH. Factors associated with propensity to aggression in Korean adolescents enrolled in a middle school. Korean J Fam Pract 2011;1:24-31. 